\title{
МОДЕЛЬ ИНСТИТУЦИОНАЛЬНОЙ СРЕДЫ СУБЪЕКТОВ МАЛОГО И СРЕДНЕГО ПРЕДПРИНИМАТЕЛЬСТВА
}

\section{(c) 2020 Родионов Дмитрий Григорьевич}

доктор экономических наук, профессор, Высшая инженерно-экономическая школа Санкт-Петербургский политехнический университет Петра Великого (СПбПУ), Россия, Санкт-Петербург E-mail: dmitry.rodionov@spbstu.ru

(C) 2020 Ялымов Сергей Вячеславович

Высшая инженерно-экономическая школа Санкт-Петербургский политехнический университет Петра Великого (СПбПУ), Россия, Санкт-Петербург

\section{(c) 2020 Конников Евгений Александрович}

кандидат экономических наук, Высшая инженерно-экономическая школа Санкт-Петербургский политехнический университет Петра Великого (СПбПУ), Россия, Санкт-Петербург

E-mail: konnikov.evgeniy@gmail.com

Сектор малого и среднего предпринимательства является одним из системообразующих в экономике России. При этом, значимость данного сектора для институциональной среды крайне дифференцирована и включает в себя множество функций социального характера. При этом институциональная среда сектора малого и среднего предпринимательства является комплексной, и функционирует в условиях многомерной системной реакции на внутренние и внешние изменения. В данной статье авторы предлагают модель институциональной среды субъектов малого и среднего предпринимательства, а также математическое выражение механизмов взаимодействия ее элементов.

Ключевые слова: сектор малого и среднего предпринимательства, МСП, институциональная среда.

Институциональная среда, как категория современной экономической науки является дискуссионной. Согласно авторам данного термина, Д.Норту и Л.Девису, под институциональной средой следует понимать совокупность основополагающих политических, социальных и юридических правил, которая образует базис для производства, обмена и распределения [1]. О.Уильямсон выражает солидарность с определением Д.Норта и Л.Дэвиса, и трактует институциональную среду как правила институционального взаимодействия, определяющие контекст экономической деятельности [2]. Американский исследователь П.Кляйн также утверждает, что именно институциональная среда формирует рамки для деятельности человека [3].

Однако, трактовка данного понятия в отечественной науке имеет свои отличия. А.Н. Олейник понимает под институциональной средой значимую для экономической деятельности характеристику внешней среды, совокупность ценностей, формальных и неформальных норм, которые влияют на соотношение стимулов в деятельности и обусловливают достижение минимального согласия между людьми [4]. Данное определение, несмотря на относительную схожесть с западными, выделяет отдельные элементы институциональной среды. Однако, в данном определении все еще недостаточно конкретики. В рамках данной работы наиболее подходящим является определение Е.С.Силовой, согласно которому институциональная среда это сложная упорядоченная система базисных и инфраструктурных экономических институтов, создающих условия для общественного воспроизводства через формирование общеобязательных правил и механизмов их поддержания, с другой стороны,- система отношений между экономическими субъектами и институтами по поводу удовлетворения потребностей экономических субъектов в институтах и институциональных механизмах [5]. Данное определение, 
помимо определения целевого вектора существования институциональной среды, определяет ее как систему экономических институтов. Таким образом, под институциональной средой субъектов малого и среднего предпринимательства (далее МСП) можно понимать системную совокупность экономических институтов и механизмов их взаимодействия в рамках процесса удовлетворения потребностей экономических субъектов.

Институциональная среда субъекта МСП может быть дифференцирована на 2 уровня - микроинституциональную среду и макроинституциональную среду. Под микроинституциональной средой субъекта МСП следует понимать совокупность динамически взаимодействующих с предприятием субъектов. При этом данное взаимодействие является билатеральным, что определяет способность субъектов МСП оказывать ответное воздействие на субъекты микроинституциональной среды, а также посредствам данного воздействия моделировать ответные реакции данных субъектов, и данному взаимодействию присуща аллелопатия, что определяет способность субъектов микроинституциональной среды оказывать как положительное, так и отрицательное воздействие на субъект МСП. Микроинституциональная среда является ограниченно управляемой, что определят способность субъектов МСП формировать ее с учетом собственных целей. Однако, полноценное управление микроинституциональной средой возможно исключительно при наличии монопольной власти конкретного субъекта МСП, что крайне несвойственно сектору МСП. Микроинституциональная среда исследовалась многими учеными. Одной из наиболее распространенных и универсальных можно выделить модель Ней- лбуффа Ибранденбургера [6], представленную на рисунке 1.

Как можно видеть на рисунке 1, горизонтальный контур отражает производственную специфику, представляя предприятие как элемент, перерабатывающий некие ресурсы, поступающие от поставщиков, и предоставляющие результаты переработки покупателям в виде готовый продукции. При этом, предприятие подвержено воздействию со стороны конкурентов и комплименторов. Данное воздействие является объективной реакцией взаимодействия данных субъектов с поставщиками и покупателями. Взаимодействие конкурентов и комплименторов с поставщиками определяет доступность ресурсов для предприятия, а взаимодействие данных субъектов с покупателями определяет спрос на готовую продукцию предприятия. При этом, данная модель не отражает способность предприятия воздействовать на какие-либо субъекты микроинституциональной среды, за исключением покупателей, что не соответствует действительности. Следовательно, данная модель должна быть дополнена совокупностью обратных связей, отражающих способность предприятия влиять на микроинституциональную среду.

Макроинституциональная среда субъекта МСП значительно более дифференцирована, и подходов к ее формализации в науке крайне много. Макроинституциональную среду можно представить как совокупность субсред, дифференцировках в соответствии с конкретным классификационным признаком, и выраженных факторами, отражающими направленное моновлияние данной субсреды на микроинституциональную среду предприятия. Отличительной особенностью макроинституциональной среды

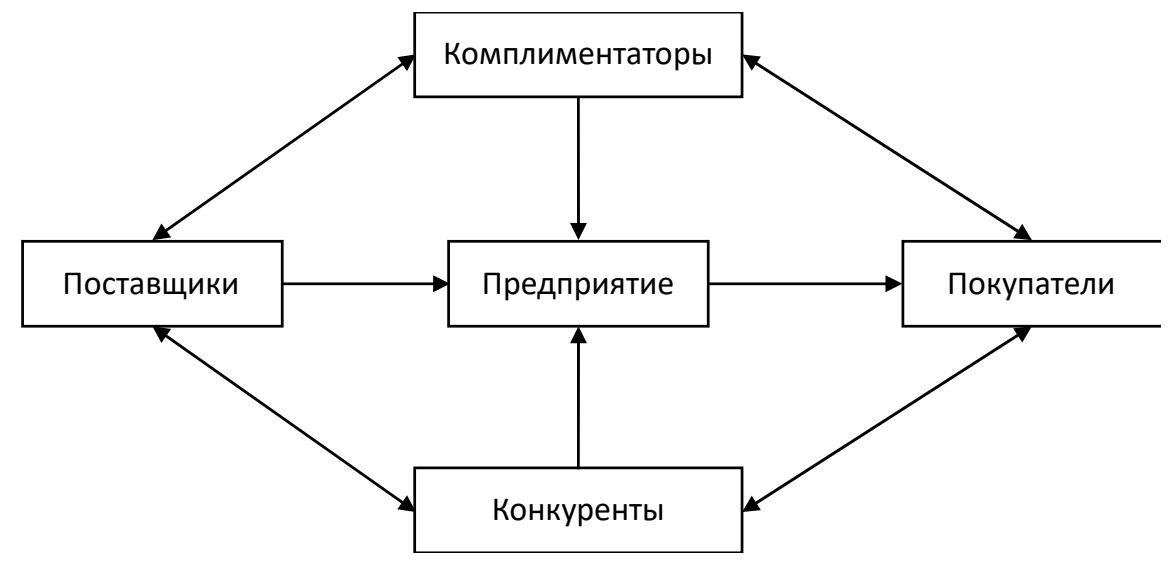

Рисунок 1. Модель микроинституциональной среды Нейлбуффа Ибранденбургера 
является отсутствие значимой обратной связи со стороны микроинституциональной среды. Факторы макроинституциональной среды оказывают влияние на микроинституциональную среду предприятия, тем самым трансформируя само предприятие, при этом субъекты микроинституциональной среды не в состояние направленно трансформировать макроинституциональную. Так как объектом является сектор МСП, следует отметить, что не все выделяемые в науке субсреды необходимо рассматривать. В частности, природно-географическая субсреда в меньшей степени оказывает влияние на субъекты МСП, в контексте трансформации инструментов их развития, что обусловлено, в первую очередь спецификой территориального распределения данных предприятий. В рамках данной работы могут быть выделены следующие субсреды макроинституциональной среду субъектов МСП:

1. Политико-правовая среда. Данная субсреда отражает влияние общегосударственных и нормативных факторов, оказывающих влияние на микроинституциональную среду субъектов МСП.

2. Социально-экономическая среда. Данная субсреда отражает влияние факторов социального характера на микроинституциональную среду субъектов МСП, что определяет ее комплексность и дифференцированность субъектов ее формирования. Вследствие данной природы, данная среда является одной из наименее про- гнозируемых, так как субъектами управления в данном случае выступают люди и социальные группы, удельный вес которых незначительный. В первую очередь данная субсреда определяет специфику потребления, что для малых и средних предприятий, в условиях рыночной экономики, является первичным.

3. Инновационная среда. Данная субсреда характеризует динамику научно-технического прогресса, склонность микроинституциональной среды к изменению в следствии инновационного развития, а также ценности данного развития для потребителей.

4. Информационная среда. Данная субсреда является наиболее вариативной и комплексной, так как она отражает содержание информационного фона и специфику моментного взаимодействия субъектов МСП с субъектами микроинституциональной среды в контексте данного информационного фона.

Выделенные субсреды формируют единую макроинституциональную среду МСП. Структура совокупной институциональной среды субъекта МСП представлена на рисунке 2.

Как можно видеть на рисунке 2, совокупное число векторов взаимодействия составляет 34 . Из них 8 векторов определяют прямое взаимодействие субъектов МСП и субъектов микроинституциональной среды $\left(\mathrm{X}_{1}-\mathrm{X}_{8}\right)$. Специфика данного взаимодействия может быть описана посредствам математических функций, подра-

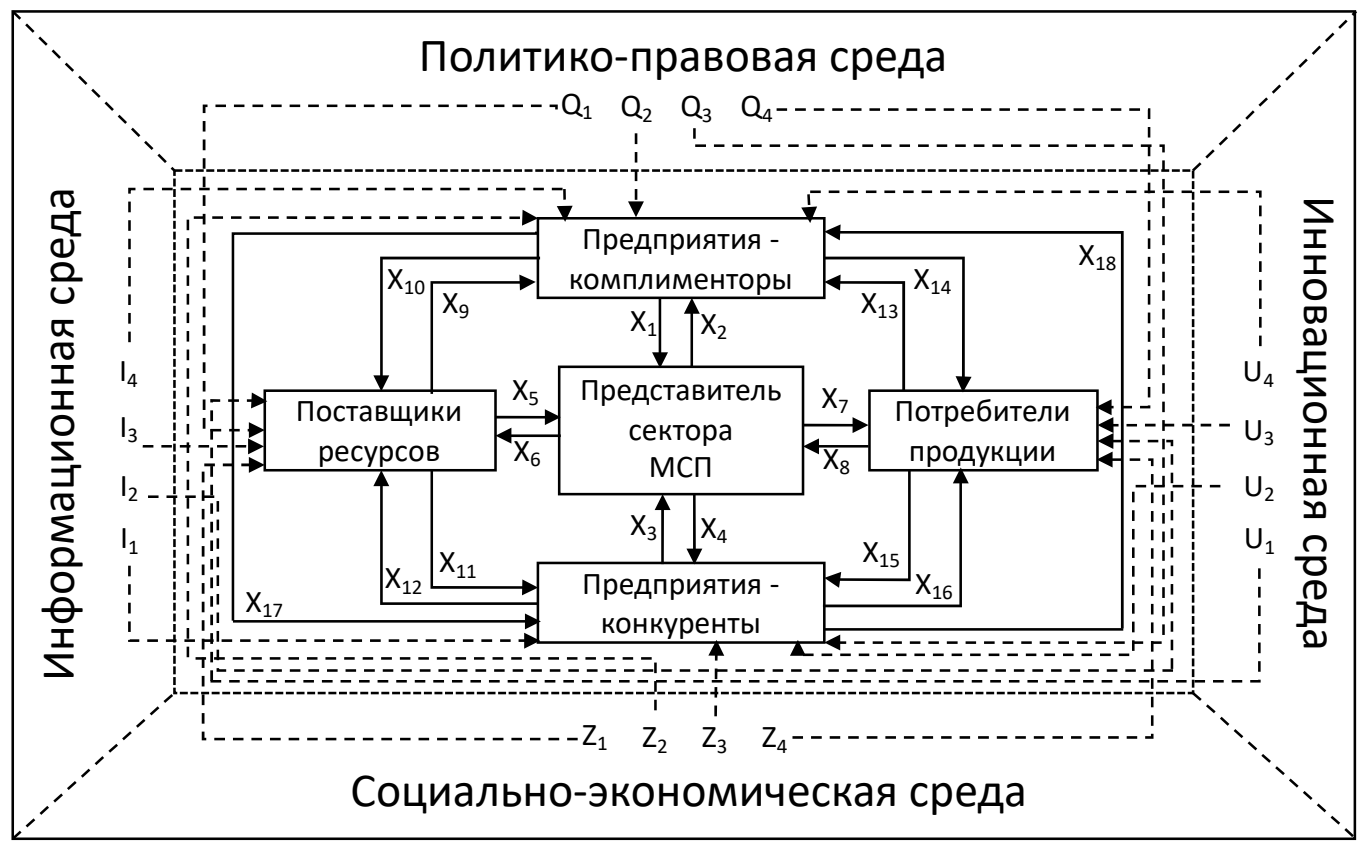

Рисунок 2. Институциональная среда субъекта МСП 
зумевающих наличие обратной связи, распределенной во времени. Взаимодействие субъектов микроинституциональной среды вне прямого воздействия на субъектов МСП описывается 10 векторами $\left(\mathrm{X}_{11}-\mathrm{X}_{18}\right)$. Данное взаимодействие имеет исключительно косвенное влияние на субъекты МСП, выражающееся в колебаниях $\mathrm{X}_{1}-\mathrm{X}_{8}$. Данные 10 выделенных связей могут быть описаны количественными переменными, названными медиаторами. Влияние макроинституциональной среды на субъектов микроинституциональной среды выражено 16 векторами, которые определяют изменения $\mathrm{X}_{11}-\mathrm{X}_{18}$. Данное влияние также может быть выражено количественными переменными, названными трансмиттерами, а их влияние на субъектов МСП может быть конвертировано посредствам математического описания их влияния на $\mathrm{X}_{11}$ $\mathrm{X}_{18}$. Таким образом описываемая система имеет трехуровневую структуру, предполагающую помимо иерархического воздействия, также и наличие горизонтальных связей. Для целей математической формализации описанной модели необходимо описать выделенные графы и определить значимость их влияния на субъектов МСП. В первую очередь необходимо математически описать взаимодействие субъектов МСП с иными субъектами микроинституциональной среды, а также определить механизмы взаимодействия субъектов микроинституциональной среды вне контекста взаимодействия с субъектами МСП. Таким образом, центральной количественной категорией на уровне микроинституциональной среды являются медиаторы.

Представленная на рисунке 2 модель институциональной среды субъекта МСП может дифференцироваться в зависимости от уровня принятия управленческих решений. В частности, на уровне конкретного предприятия (субъекта МСП) в качестве конкурентов могут рассматриваться в первую очередь другие субъекты МСП или крупные предприятия, производящие конкурентные товары и услуги. На уровне муниципалитета в качестве субъекта МСП может рассматриваться совокупность предприятий, производящих товары и оказывающих услуги в рамках конкретного муниципального образования, а в качестве конкурентов - предприятия, производящие конкурентные товары или товары заменители и оказывающие услуги в иных муниципальных округах. На уровне региона принцип масштабирования сохраняется.
На макроуровне (уровне государства) МСП рассматривается как единый сектор, а в качестве конкурентов выступает крупный бизнес и иностранный сектор. Таким образом, описываемая модель является универсальной, однако она требует конкретизации относительно субъекта управления. Следовательно, выводы, которые могут быть сформулированы на основе динамического системного анализа данной модели, значимо дифференцируются в зависимости от объекта, субъекта и цели анализа.

В первую очередь рассмотрим связи между представителями сектора МСП и потребителями производимых ими товаров, работ или оказываемых услуг (далее ТРУ), выраженные в векторах $\mathrm{X}_{7}$ и $\mathrm{X}_{8}$. Вектор $\mathrm{X}_{7}$ описывает поток благ и/или информации (в том случае если информация не является благом, производимым представителем МСП) от субъекта МСП к потребителю. Данный поток может быть назван потенциальным предложением ТРУ. На уровне конкретного предприятия данный поток выражается потенциально возможным объемом реализации ТРу данного предприятия, измеренным в денежных единицах, в то время как на муниципальном и региональном уровне данный показатель отражает потенциально возможный объем реализации ТРУ совокупности субъектов МСП (возможно в отраслевом разрезе), а на макроуровне (федеральном уровне) - потенциально возможный оборот всего сектора МСП (также возможно в отраслевом разрезе). Динамика данного показателя определяется 2 базовыми характеристиками субъекта МСП:

1. Производственными возможностями. Данная совокупность характеристик субъекта МСП объединяет в себе всю специфику ресурсного обеспечения предприятия, и математически характеризуется в первую очередь предельным объемом производства.

2. Спросом на ТРУ. Данная комплексная характеристика внешней среды является определяющей, так как она количественно отражает склонность рыночных субъектов потреблять ТРУ субъекта МСП. Данная характеристика внешней среды в рамках представленной схемы выражена вектором $\mathrm{X}_{8}$.

Отражая потенциальный спрос потребителей на ТРУ субъекта МСП, вектор $\mathrm{X}_{8}$ измеряется в денежных единицах и отражает платежеспособную потребность субъектов рынка в ТРУ субъекта МСП. Данный показатель также мас- 
штабируется в зависимости от объекта и целей исследования. Взаимодействие показателей $\mathrm{X}_{7}$ и $\mathrm{X}_{8}$ описывается посредством законов спроса и предложения. В условиях идеального баланса внешней среды отношение данных показателей должно стремиться к 1, а производная по функции, описывающая изменение $\mathrm{X}_{8}$, должна быть не отрицательной. Таким образом, $\mathrm{X}_{7}$ может рассматриваться как зависимый показателей, стремящийся к $\mathrm{X}_{8}$. Следовательно, математически описать целевое изменение данных показателей можно посредствам следующих правил:

$$
\frac{X_{8}}{X_{7}} \rightarrow 1 ; \Delta X_{8} \geq 0 ; \Delta X_{7} \rightarrow \Delta X_{8}
$$

Где:

$\mathrm{X}_{7}$ - потенциальное предложение ТРУ представителем МСП;

$\mathrm{X}_{8}$ - потенциальный спрос на ТРУ субъекта МСП со стороны потребителей;

$\Delta \mathrm{X}_{8}$ - изменение потенциального спроса на ТРУ субъекта МСП во времени.

Данные математические правила являются базовыми для описания изменения состояния среды субъекта МСП (или сектора МСП в целом), а следовательно, принятия управленческих решений относительно построения стратегии развития. При этом одним из ключевых факторов изменения предложения является трансформация структуры ресурсного обеспечения предприятия, в первую очередь связанная с поставщиками, а ключевым фактором изменения спроса является трансформация отношений конкурентных преимуществ ТРУ субъекта МСП. Данный факт порождает необходимость детального описания взаимодействия остальных субъектов микроинституциональной среды.

Далее рассмотрим вектора взаимодействие субъекта МСП и предприятий конкурентов. В данном случае конкурентами являются предприятия, ТРУ которых могут удовлетворять спрос на ТРУ субъекта МСП, тем самые снижая его. Данное взаимодействие описывается показателями $\mathrm{X}_{4}$ и $\mathrm{X}_{3}$. Данные показатели, в отличии от $\mathrm{X}_{7}$ и $\mathrm{X}_{8}$, отражают не поток конкретных благ, выраженных в денежных единицах, а отражают в первую очередь соотношение сравнительных характеристик ТРУ, являющихся предметом конкуренции. При этом данные показатели являются динамическими, так как моментное состояние конкурентоспособности ТРУ субъекта МСП в состоянии влиять на спрос исключительно ограни- ченно. Таким образом, под $\mathrm{X}_{4}$ можно понимать индекс изменения конкурентоспособности ТРУ субъекта МСП за период времени. Данный показатель является ранговым, и измеряется в условных единицах, распределенных от 0 до 2 , где 0 - абсолютное снижение конкурентоспособности, а 2 - абсолютный рост конкурентоспособности. При $\mathrm{X}_{4}$ равном единице можно говорить о сохранении уровня конкурентоспособности ТРУ субъекта МСП. Идентичную структуру имеет $\mathrm{X}_{3}$, отражающий изменение конкурентоспособности ТРУ предприятий-конкурентов субъекта МСП. Надо отметить, что данные показатели не являются обратными друг другу, так как изменение спроса может носить однонаправленный характер по отношению как к ТРУ субъекта МСП, так и к ТРУ конкурентов, что приводит к изменению совокупного спроса. Отношение $\mathrm{X}_{4} \mathrm{~K} \mathrm{X}_{3}$ отражает сравнительный прирост конкурентоспособности ТРУ субъекта МСП. Результатом повышения $\mathrm{X}_{4}$ становится увеличение потенциального спроса, выраженного $\Delta \mathrm{X}_{8}$. Для конкурентов субъекта МСП потенциальный спрос выражается показателем $\mathrm{X}_{15}$, следовательно его изменение $-\Delta \mathrm{X}_{15}$. Совокупная платежеспособная потребность потребителей в тех или иных ТРУ формируется как сумма спроса на ТРУ субъекта МСП и спроса на ТРУ его конкурентов. Таким образом изменение совокупного спроса на ТРУ за счет изменения конкурентоспособности продукции может быть рассчитано посредствам следующего выражения:

Без учета изменения совокупного спроса:

$$
\Delta X_{c}^{k}=\left(X_{8} * \frac{X_{4}}{X_{3}}+X_{15} * \frac{X_{3}}{X_{4}}\right)-X_{c}
$$

С учетом изменения совокупного спроса:

$$
\Delta X_{c}^{\mathrm{KOH}}=\left(X_{8} * X_{4}+X_{15} * X_{3}\right)-X_{c}
$$

Где:

$\mathrm{X}_{\mathrm{c}}$ - совокупный спрос на конкретные ТРУ;

$\Delta \mathrm{X}_{\mathrm{C}}^{\mathrm{KoH}}$ - изменение совокупного спроса за счет изменения конкурентоспособности ТРУ субъекта МСП;

$\mathrm{X}_{4}$ - индекс изменения конкурентоспособности ТРУ субъекта МСП;

$\mathrm{X}_{3}$ - индекс изменения конкурентоспособности ТРУ предприятий-конкурентов субъекта МСП.

В данном случае дифференциация данного 
математического правила определяется необходимостью дифференциации возможности учета направленной трансформации спроса, которая может быть вызвана множеством причин. В качестве примера данной направленной трансформации можно привести спрос на одноразовые медицинские маски в условиях пандемии. Потенциальное предложение ТРУ предприятий конкурентов субъекта МСП выражено показателем $\mathrm{X}_{16}$. Данный показатель сущностно идентичен показателю $\mathrm{X}_{7}$, но при этом не является значимым с исследовательской точки зрения. В связи с этим, в дальнейшем данный показатель не рассматривается.

Далее рассмотрим взаимодействие субъектов МСП и предприятий-комплименторов. Данное взаимодействие выражается показателями $\mathrm{X}_{1}$ и $\mathrm{X}_{2}$. Данные показатели оказывают косвенное воздействие на состояние субъекта МСП в институциональной среде, так как предприятия-комплименторы не являются напрямую конкурирующими субъектами по отношению к исследуемому субъекту МСП, а следовательно не формируют какую-либо часть уникального спроса (не являются элементом $\mathrm{X}_{\mathrm{c}}$ ). При этом, за счет влияния предприятийкомплименторов спрос на ТРУ субъектов МСП может увеличиваться или уменьшаться. Данное влияние выражено показателем $\mathrm{X}_{1}$. Данный показатель отражает изменение конкурентоспособности ТРУ субъекта МСП за счет влияния предприятий-комплименторов. При этом, показатель $\mathrm{X}_{2}$, в свою очередь является обратным, и отражает изменение конкурентоспособности ТРУ предприятий-комплименторов субъекта МСП. Так как предприятия-комплименторы могут также выступать представителями МСП, данный показатель может одновременно выступать в качестве показателя $\mathrm{X}_{1}$ по отношению к ним. В рамках данного исследования, данный показатель не рассматривается в качестве целевого. Идентичный тезис справедлив для показателей $\mathrm{X}_{14}$ и $\mathrm{X}_{13}$, так как они отражают потенциальное предложение и потенциальный спрос на ТРУ предприятий-комплименторов субъекта МСП. Показатель $\mathrm{X}_{1}$ в свою очередь выступает мультипликатором $\mathrm{X}_{8}$. Данный показатель также является ранговым, и измеряется в диапазоне от 0 до 2 , где отсутствие какого-либо значимого изменения выражается единичным значением. Помимо влияния на субъекты МСП, предприятия-комплименторы взаимодейству- ют с его конкурентами. Данное взаимодействие выражается посредствам показателей $\mathrm{X}_{17}$ и $\mathrm{X}_{18}$. Показатель $\mathrm{X}_{17}$ в свою очередь отражает изменение конкурентоспособности ТРУ предприятийконкурентов субъекта МСП за счет воздействия предприятий-комплименторов. Природа данного показателя во многом идентично показателю $\mathrm{X}_{1}$, вследствие чего данный показатель является обратным мультипликатором $\mathrm{X}_{15}$. Таким образом, изменение совокупного спроса на ТРУ за счет влияния предприятий комплименторов может быть рассчитано следующим образом:

$$
\Delta X_{c}^{\text {ком }}=\left(X_{8} * X_{1}+X_{15} / X_{17}\right)-X_{c}
$$

Где:

$\Delta \mathrm{X}_{\mathrm{c}}^{\mathrm{KOH}}$ - изменение совокупного спроса за счет влияния предприятий-комплименторов;

$\mathrm{X}_{1}$ - индекс изменения конкурентоспособности ТРУ субъекта МСП за счет влияния предприятий-комплименторов;

$\mathrm{X}_{17}$ - индекс изменения конкурентоспособности ТРУ предприятий-конкурентов субъекта МСП за счет влияния предприятийкомплименторов.

Сумма приведённых изменений формирует совокупное изменение спроса на ТРУ. Индикатором направленного изменения институциональной среды, относительно субъекта МСП, является скорость изменения спроса на ТРУ субъекта МСП относительно скорости изменения спроса на ТРУ предприятий конкурентов. Данный индикатор может быть рассчитан как изменение отношения спроса на ТРУ субъекта МСП к спросу на товары предприятий-конкурентов:

$$
\begin{gathered}
F_{0}=\frac{X_{8}}{X_{15}} \\
F_{1}=\frac{X_{8} * \frac{X_{4} * X_{1}}{X_{3}}}{X_{15} * \frac{X_{3}}{X_{4} * X_{17}}} \\
\Delta F_{\text {общ }}=\frac{X_{8} * \frac{X_{4} * X_{1}}{X_{3}}}{X_{15} * \frac{X_{3}}{X_{4} * X_{17}}}-\frac{X_{8}}{X_{15}}
\end{gathered}
$$




$$
\begin{aligned}
& \Delta F_{X_{8}}=\frac{X_{8} * \frac{X_{4} * X_{1}}{X_{3}}-X_{8}}{X_{15} * \frac{X_{3}}{X_{4} * X_{17}}-X_{15}} * \ln \frac{X_{15} * \frac{X_{3}}{X_{4} * X_{17}}}{X_{15}} \\
& \Delta F_{X_{15}}=\Delta F_{\text {общ }}-\Delta F_{X_{8}}
\end{aligned}
$$

Где:

$\mathrm{F}_{1}$ - индикатор отношения спроса в отчетном периоде;

$\mathrm{F}_{0}$ - индикатор отношения спроса в базисном периоде;

$\Delta \mathrm{F}_{\text {общ }}-$ совокупное изменение индикатора отношения спроса за отчетный период;

$\Delta \mathrm{F}_{\mathrm{X} 8}$ - изменение индикатора отношения спроса за отчетный период за счет изменения спроса на ТРУ субъекта МСП;

$\Delta \mathrm{F}_{\mathrm{X} 15}$ - изменение индикатора отношения спроса за отчетный период за счет изменения спроса на ТРУ предприятий конкурентов.

В данном случае ключевыми аналитическими индикаторами изменения состояния институциональной среды субъекта МСП являются $\Delta \mathrm{F}_{\mathrm{X} 8}$ и $\Delta \mathrm{F}_{\mathrm{X} 15}$, в то время как ключевыми медиаторами изменения данных индикаторов выступают описанные ранее мультипликаторы. Данные мультипликаторы могу быть условно обозначены как $\mathrm{M}_{\mathrm{X8}}$ и $\mathrm{M}_{\mathrm{X} 15}$ :

$$
\begin{aligned}
& M_{X_{8}}=\frac{X_{4} * X_{1}}{X_{3}} \\
& M_{X_{15}}=\frac{X_{3}}{X_{4} * X_{17}}
\end{aligned}
$$

\section{Где:}

$\mathrm{M}_{\mathrm{X} 8}$ - медиатор спроса на ТРУ предприятия МСП;

$\mathrm{M}_{\mathrm{X} 15}$ - медиатор спроса на ТРУ предприятийконкурентов.

Как было установлено ранее, динамика спроса определяет динамику предложения. Однако, предложение также зависит и от специфики ресурсного обеспечения. Данная специфика проявляется в связях с поставщиками, которые выражены в первую очередь показателями $\mathrm{X}_{5}$ и $\mathrm{X}_{6}$, отражающими предложение и спрос на ресурсы, со стороны субъекта МСП. Показатель $\mathrm{X}_{6}$, отражающий спрос на ресурсы, является основой формирования потенциального предложения предприятия, скорректированного на коэффициент конверсии, отражающий долю ресурсов, образующую готовую продукцию. Предложение ресурсов в свою очередь формируется как следствие взаимодействия поставщиков с иными субъектами микроинституциональной среды, выраженным показателями $\mathrm{X}_{11}$ (потенциальное предложение ресурсов для производства ТРУ конкурентов субъекта МСП), $\mathrm{X}_{12}$ (потенциальный спрос на ресурсы для производства ТРУ со стороны конкурентов субъекта МСП) и $\mathrm{X}_{10}$ (индекс изменения воздействия комплименторов на поставщиков ресурсов субъекта МСП). В рамках данной работы рассматривается исключительно конкурентные рынки, что определяет наличие равного доступа к ресурсам со стороны участников рынка. Таким образом, потенциальный спрос на ресурсы со стороны предприятийконкурентов зависим в первую очередь от изменения спроса на ТРУ предприятий конкурентов, которое определяется посредством $\mathrm{M}_{\mathrm{X} 15}$. Также необходимо учитывать влияние предприятийкомплиментов, выраженное показателем $\mathrm{X}_{10}$. В качестве показателя, отражающего изменение структурны ресурсного обеспечения предприятия, можно использовать индекс прироста предложения:

$$
P=\frac{X_{5} *\left(M_{X_{8}}-M_{X_{15}}+X_{10}\right)}{X_{5}}
$$

Где:

P - индекс прироста предложения ресурсов.

Динамика данного показателя отражает динамику доступности ресурсов. Снижение данного показателя свидетельствует о потенциальном снижении предложения относительно роста спроса, что может спровоцировать негативные последствия для субъектов МСП.

Выделенные показатели отражают исключительно состояние микроинституциональной среды. При этом факторами, провоцирующими изменение данных показателей, являются факторы макроинституциональной среды, представленные в виде четырех субсред. Влияние каждой субсреды на каждый из выделенных показателей выражено совокупностью частных факторов, которые также могут быть выражены комплексами показателей. Следовательно, каждый из источников медиирования может быть определен посредствам расчета функционального воздействия со стороны показателей 
макроинституциональной среды. Данные показатели могут быть названы трансмиттерами, так как выступают в данном случае в качестве медиаторов самих медиаторов. Функциональная связь между составными показателями медиаторов и трансмиттерами может быть представлена посредствам следующих уравнений:

$$
\begin{aligned}
& X_{4}=f\left(Z_{3.1} ; U_{2.1} ; Q_{3.1} ; I_{1.1}\right) \\
& X_{3}=f\left(Z_{3.2} ; U_{2.2} ; Q_{3.2} ; I_{1.2}\right) \\
& X_{1}=f\left(Z_{2.1} ; U_{4.1} ; Q_{2.1} ; I_{4.1}\right) \\
& X_{17}=f\left(Z_{2.2} ; U_{4.2} ; Q_{2.2} ; I_{4.2}\right) \\
& X_{10}=f\left(Z_{2.3} ; U_{4.3} ; Q_{2.3} ; I_{4.3}\right)
\end{aligned}
$$

Где:

$Z_{3.1}$ - интегральный показатель влияния социально-экономической среды на изменение конкурентоспособности ТРУ субъекта МСП;

$\mathrm{U}_{2.1}$ - интегральный показатель влияния инновационной среды на изменение конкурентоспособности ТРУ субъекта МСП;

$\mathrm{Q}_{3.1}$ - интегральный показатель влияния политико-правовой среды на изменение конкурентоспособности ТРУ субъекта МСП;

$\mathrm{I}_{1.1}$ - интегральный показатель влияния информационной среды на изменение конкурентоспособности ТРУ субъекта МСП;

$\mathrm{Z}_{3.2}$ - интегральный показатель влияния социально-экономической среды на изменение конкурентоспособности ТРУ предприятийконкурентов субъекта МСП;

$\mathrm{U}_{2.2}$ - интегральный показатель влияния инновационной среды на изменение конкурентоспособности ТРУ предприятий-конкурентов субъекта МСП;

$\mathrm{Q}_{3.2}$ - интегральный показатель влияния политико-правовой среды на изменение конкурентоспособности ТРУ предприятийконкурентов субъекта МСП;

$\mathrm{I}_{1.2}$ - интегральный показатель влияния информационной среды на изменение конкурентоспособности ТРУ предприятий-конкурентов субъекта МСП;

$\mathrm{Z}_{2.1}$ - интегральный показатель влияния социально-экономической среды на изменение конкурентоспособности ТРУ субъекта МСП за счет влияния предприятий-комплименторов;

$\mathrm{U}_{4.1}$ - интегральный показатель влияния инновационной среды на изменение конкурентоспособности ТРУ субъекта МСП за счет влияния предприятий-комплименторов;

$\mathrm{Q}_{2.1}$ - интегральный показатель влияния политико-правовой среды на изменение конкурентоспособности ТРУ субъекта МСП за счет влияния предприятий-комплименторов;

$\mathrm{I}_{4.1}$ - интегральный показатель влияния информационной среды на изменение конкурентоспособности ТРУ субъекта МСП за счет влияния предприятий-комплименторов;

$\mathrm{Z}_{2.2}$ - интегральный показатель влияния социально-экономической среды на изменение конкурентоспособности ТРУ предприятийконкурентов субъекта МСП за счет влияния предприятий-комплименторов;

$\mathrm{U}_{4.2}$ - интегральный показатель влияния инновационной среды на изменение конкурентоспособности ТРУ предприятий-конкурентов субъекта МСП за счет влияния предприятийкомплименторов;

$\mathrm{Q}_{2.2}$ - интегральный показатель влияния политико-правовой среды на изменение конкурентоспособности ТРУ предприятийконкурентов субъекта МСП за счет влияния предприятий-комплименторов;

$\mathrm{I}_{4.2}$ - интегральный показатель влияния информационной среды на изменение конкурентоспособности ТРУ предприятий-конкурентов субъекта МСП за счет влияния предприятийкомплименторов;

$\mathrm{Z}_{2.3}$ - интегральный показатель влияния социально-экономической среды на изменение воздействия комплименторов на поставщиков ресурсов субъекта МСП;

$\mathrm{U}_{4.3}$ - интегральный показатель влияния инновационной среды на изменение воздействия комплименторов на поставщиков ресурсов субъекта МСП;

$\mathrm{Q}_{2.3}$ - интегральный показатель влияния политико-правовой среды на изменение воздействия комплименторов на поставщиков ресурсов субъекта МСП;

$\mathrm{I}_{4.3}$ - интегральный показатель влияния информационной среды на изменение воздействия комплименторов на поставщиков ресурсов субъекта МСП.

Представленный комплекс показателей является производным от факторов макроинституциональной среды МСП. Данные факторы являются достаточно комплексными и неоднородными, что определяет необходи- 
мость их трансформации в комплексы условнообъективных статистических или экспертных показателей с прозрачной процедурой определения. Определение сил влияния и векторов воздействия приведенных показателей на фор-

\section{Библиографический список}

1. Davis L., North D. Institutional Change and American Economic Growth. Cambridge. 1971. - P. 6.

2. Уильямсон О.И. Экономические институты капитализма. Фирмы, рынки, «отношенческая» контрактация / Науч. ред. и вступ, статья В. С. Катькало.-Спб.: Лениздат; CEV Press, 1996. - 702 с.

3. Klein, Peter G., New Institutional Economics. July 1998. Экономический факультет университета штата Джорджия, США.

4. Олейник А.Н., Институциональная экономика: учеб. пособие / А. Н. Олейник. М.: ИНФРА-М, 2000.

5. Силова Е.С. Качество институциональной среды и его влияние на экономический рост // Дис. канд. экон. наук. Челябинск, 2007.

6. Ханс Хершген, Александр Макаров. Маркетинговая ориентация как фактор успеха предприятий инвестиционного сектора экономики // Практический маркетинг. № 102004 URL: https://www.cfin.ru/press/ practical/2004-10/01.shtml\#_ftn3 (дата обращения: 15.08.2020) 